\title{
Sichere Stromversorgung bei hohen Anteilen volatiler erneuerbarer Energien
}

\section{Was kann ein Strommarkt 2.0 leisten?}

In den letzten Jahren wird vermehrt die Sorge geäußert, dass die Energiewende die Sicherheit der Stromversorgung in Deutschland langfristig durch Engpässe und Stromausfälle gefährden könnte. Während der Ausbau von volatiler Stromerzeugung aus Wind- und Sonnenenergie mit hohem Tempo voranschreitet, sinken die Anreize, in konventionelle Reservekraftwerke zu investieren. Auf diese Befürchtungen reagiert die Bundesregierung nun mit einem energiepolitischen Reformpaket für einen sogenannten „Strommarkt 2.0“. Aber kann damit die Stromversorgung tatsächlich nachhaltig gesichert werden?

In Sachen Stromversorgungssicherheit geben insbesondere die Ankündigungen einiger Kraftwerksbetreiber, ihre bestehenden Kraftwerke vom Netz zu nehmen und bereits geplante neue Kraftwerke nicht mehr zu bauen, Grund zur Sorge. ${ }^{1}$ Ursachen sind sinkende Börsenstrompreise und mangelnde Auslastung, die in den letzten Jahren insbesondere den Betrieb von Gaskraftwerken zunehmend unrentabel gemacht haben. Bislang ist jedoch umstritten, ob es sich bei diesen Entwicklungen um ein grundsätzliches, strukturelles Problem handelt, das im Zuge der Energiewende auftritt. So können die gegenwärtig niedrigen Börsenstrompreise zwar auch darauf zurückgeführt werden, dass staatlich geförderter Strom aus erneuerbaren Energiequellen mit Grenzkosten von Null auf den Markt drängt. Maßgeblicher haben bislang jedoch der Verfall von $\mathrm{CO}_{2}$ - und Rohstoffpreisen sowie Überkapazitäten in der Stromerzeugung zu sinkenden Börsenstrompreisen beigetragen. ${ }^{2}$ Trotzdem wird befürchtet, dass die Energiewende mittelfristig vor einem Versorgungssicherheitsproblem stehen könnte, zumal bis 2022 auch die verbliebenen Kernkraftwerke schrittweise abgeschaltet werden.

\section{Gesetzentwurf für einen „Strommarkt 2.0“}

Als Reaktion auf diese Befürchtungen und massiven Branchendruck hat das zuständige Bundesministerium

1 M. Balser, M. Bauchmüller: RWE könnte weitere Kraftwerke stilllegen, in: Süddeutsche Zeitung vom 12.8.2014; o.V.: Energieversorger wollen 57 Kraftwerke abschalten, in: Die Welt vom 24.8.2015.

2 Vgl. etwa T. Kallabis, C. Pape, C. Weber: A parsimonious fundamental model for wholesale electricity markets - Analysis of the plunge in German future prices, EWL Working Paper, Nr. 4/15, Universität Duisburg-Essen 2015. für Wirtschaft und Energie (BMWi) im letzten Jahr nach langer und kontroverser Debatte ein Konzept für einen ertüchtigten „Strommarkt 2.0“ vorgelegt. ${ }^{3}$ Der entsprechende Gesetzentwurf der Bundesregierung wird gegenwärtig im Bundestag beraten. ${ }^{4}$ Dieser „Strommarkt 2.0“ beruht dabei im Kern auf zwei Säulen. Zum einen setzt der Entwurf explizit auf die Stärkung der Marktmechanismen (§ 1a Energiewirtschaftsgesetz, EnWG-E). Die Höhe der Strompreise im Großhandel (Termin-, Day-Head-, Intraday-, Day-After- und Regelleistungsmärkte) soll (weiterhin) nicht regulatorisch beschränkt werden. Die Bilanzkreisverantwortlichen sollen als Mittler der Markttransaktionen mehr Verantwortung und durch Pönale Anreize zur Sicherung der Stromversorgung erhalten. Kleinen, dezentralen Anbietern von flexibler Stromerzeugung und -nachfrage soll der Zugang zu den Regelenergiemärkten erleichtert werden. Zudem soll grundsätzlich die Transparenz der Strommärkte erhöht werden. Gleichzeitig soll der Strommarkt aber auch durch mehrere Kraftwerksreserven abgesichert werden: eine gestärkte Netzreserve ( $\S 13 d$ EnWG-E) sowie eine neu geschaffene Kapazitätsreserve ( $(13 e$ EnWG-E). Außerdem werden die ältesten Braunkohlekraftwerke zur Erreichung der nationalen Klimaschutzziele vorläufig stillgelegt und in eine Klimareserve überführt (§ $13 \mathrm{~g}$ EnWG-E). Kraftwerke, die diesen drei Reserven zugerechnet werden, müssen in Einsatzbereitschaft gehalten werden, dürfen jedoch

3 BMWi: Ein Strommarkt für die Energiewende. Ergebnispapier des Bundesministeriums für Wirtschaft und Energie (Weißbuch), Berlin 2015, http://www.bmwi.de/BMWi/Redaktion/PDF/Publikationen/weissbuch, property=pdf, bereich=bmwi2012, sprache=de,rwb=true. pdf.

4 Entwurf eines Gesetzes zur Weiterentwicklung des Strommarktes (Strommarktgesetz), Bundestagsdrucksache 18/7317, http://dip21. bundestag.de/dip21/btd/18/073/1807317.pdf. 
nur im Engpassfall und auf Anweisung der Netzbetreiber Strom einspeisen. Für die Bereitstellung dieser Kapazität erhalten die Kraftwerksbetreiber eine Ausgleichszahlung. Zudem soll die zukünftige Versorgungssicherheit durch ein Monitoring fortlaufend überwacht werden (§ 51 EnWG-E).

Insgesamt setzt das BMWi zur Sicherung der Stromversorgung zukünftig also auf eine Doppelstrategie. Grundsätzlich soll das Vertrauen in die Funktionsfähigkeit des Marktes gestärkt werden. In Anbetracht der Unsicherheit über die tatsächliche zukünftige Entwicklung von Stromangebot und -nachfrage wird mit den Reserven jedoch ein zusätzliches Fangnetz aufgespannt, um die Stromversorgung zu sichern. Von einer grundlegenderen Reform des Strommarktes, insbesondere der Schaffung völlig neuer „Kapazitätsmärkte“, wie sie weite Teile der Energiewirtschaft gefordert hatten, ${ }^{5}$ wurde hingegen Abstand genommen. Es bleibt die Frage offen, ob ein so abgesicherter „Strommarkt 2.0“ dazu taugt, die Versorgungssicherheit mittel- und langfristig in nachhaltiger Weise zu gewährleisten. So hat die Monopolkommission bereits Kritik am Gesetzentwurf geäußert. ${ }^{6}$ Grundsätzlich stellt sie infrage, ob (in Knappheitssituationen extreme) Preisspitzen auf Strommärkten politisch durchgehalten werden können und unter Marktmachtgesichtspunkten kontrollierbar bleiben. ${ }^{7}$ Nur dann könne ein "Strommarkt 2.0“ marktgerecht funktionieren. Zudem sei der Ansatz einer Kapazitätsreserve durch die diversen administrativen Vorgaben allokativ ineffizient und anfällig für Marktmacht und politische Einflussnahme. Die Monopolkommission plädiert daher dafür, Kapazitätsreserven nur für eine Übergangsphase zu nutzen und nach spätestens zehn Jahren wieder abzuschaffen.

\section{Nachhaltige Sicherung der Stromversorgung}

Um den Vorschlag des BMWi wie auch die Kritik daran einordnen und bewerten zu können, ist zunächst ein Blick auf die grundlegenden Voraussetzungen für eine nach-

5 Für einen Überblick vgl. etwa W.-P. Schill, J. Diekmann: Die Kontroverse um Kapazitätsmechanismen für den deutschen Strommarkt, DIW Roundup 5/2014, http://www.diw.de/de/diw_01.c.434277.de/ presse/diw_roundup/die_kontroverse_um_kapazitaetsmechanismen_ fuer_den_deutschen_strommarkt.html.

6 Monopolkommission: Energie 2015: Ein wettbewerbliches Marktdesign für die Energiewende. Sondergutachten 71, 2015, http://www. monopolkommission.de/images/PDF/SG/s71_volltext.pdf.

7 So auch U. Leprich: Versorgungssicherheit im Spannungsfeld von Märkten und Regulierung - zum Design des zukünftigen Stromsystems. Impulsvortrag im Rahmen der 8. Eberbacher Klostergespräche zu ökonomischen Grundsatzfragen der Transformation des Energiesystems, 12.10.2015, https://www.htwsaar.de/wiwi/fakultaet/ personen/profile/leprich.uwe/publikationen/LeprichKlosterEberbach12. Oktober2015.pdf.
Dr. Paul Lehmann leitet die Forschungsgruppe Energieökonomik im Department Ökonomie am Helmholtz-Zentrum für Umweltforschung - UFZ in Leipzig.

Prof. Dr. Erik Gawel ist Direktor des Instituts für Infrastruktur und Ressourcenmanagement der Universität Leipzig und Leiter des Departments Ökonomie am UFZ.

Klaas Korte, Dipl.-Ökonom, ist wissenschaftlicher Mitarbeiter des Departments Ökonomie am UFZ.

Matthias Reeg, Dipl.-Wirtschaftsingenieur, ist wissenschaftlicher Mitarbeiter des Instituts für Technische Thermodynamik am Deutschen Luft- und Raumfahrtzentrum (DLR) in Stuttgart.

Dr. Dominik Schober betreut den Forschungsschwerpunkt Wettbewerb und Regulierung in Netzindustrien am Zentrum für Europäische Wirtschaftsforschung (ZEW) in Mannheim.

haltige Sicherung der Stromversorgung sinnvoll. ${ }^{8}$ Diese können in vier Thesen zusammengefasst werden:

1. Alle Marktteilnehmer können Beiträge zur Versorgungssicherheit leisten! Versorgungssicherheit im Stromversorgungssystem wird häufig als die dauerhafte und unterbrechungsfreie Deckung einer als gegeben angenommenen Stromnachfrage in einem Versorgungsgebiet definiert. ${ }^{9}$ In diesem Sinne ist die Versorgungssicherheit insbesondere durch die Stromerzeuger zu gewährleisten. Diese Definition verengt die Diskussion um Versorgungssicherheit jedoch stark. Versorgungssicherheit kann nicht nur durch den Bau oder Weiterbetrieb von Kraftwerken gewährleistet werden. Vielmehr können prinzipiell alle Akteure im Stromversorgungssystem - Betreiber von konventionellen

8 Vgl. ausführlicher M. Reeg et al.: Kapazitätsmechanismen als Rettungsschirm der Energiewende? Zur Versorgungssicherheit bei hohen Anteilen fluktuierender erneuerbarer Energien im Stromsystem, ENERGY-TRANS Discussion Paper, Nr. 1/2015, https://www.energytrans.de/925_1454.php.

9 Vgl. BMWi: Monitoring-Bericht des Bundesministeriums für Wirtschaft und Technologie nach § 51 EnWG zur Versorgungssicherheit im Bereich der leitungsgebundenen Versorgung mit Elektrizität, Berlin 2012. 
Kraftwerken, Erneuerbaren-Energie-Anlagen, Netzen und Speichern sowie Stromverbraucher - Beiträge zur Versorgungssicherheit leisten. Vor diesem Hintergrund sollte Versorgungssicherheit als der permanente Ausgleich von Stromangebot und -nachfrage unter Einbeziehung aller möglichen Handlungsoptionen der Marktakteure verstanden werden. $^{10}$ Versorgungssicherheit bezieht sich dabei zunächst auf die Fähigkeit des Stromversorgungssystems, die maximal erwartete Spitzenlast (auch unter Berücksichtigung möglicher Reduktions- und Flexibilisierungspotenziale der Nachfrage) durch gesicherte Erzeugung decken zu können (Angemessenheit der Stromversorgung)..$^{11}$ Darüber hinaus müssen jedoch auch ausreichend flexible Kapazitäten vorgehalten werden, um auf kurzfristige, unerwartete Störungen im System reagieren und die Netzstabilität idealerweise jederzeit gewährleisten zu können (Stabilität der Stromversorgung). ${ }^{12}$

2. Die Versorgungssicherheit hängt nicht allein an der Energiewende! Zweifellos fordern die Energiewende und insbesondere der massive Ausbau der Stromerzeugung aus fluktuierender und nur bedingt prognostizierbarer Wind- und Sonnenenergie die Gewährleistung der Versorgungssicherheit zusätzlich heraus. So müssen die steuerbaren (konventionellen) Kraftwerke ihre Erzeugung nicht mehr primär an den Verlauf der Stromnachfrage anpassen. Vielmehr müssen sie vor allem die Residuallast decken, also nur den Teil der Stromnachfrage, der nicht durch Strom aus erneuerbaren Energiequellen befriedigt werden kann. Dafür bedarf es in größerem Umfang als bisher flexibler Kapazitäten im Stromsystem. ${ }^{13}$ Gleichzeitig führt die Erneuerbaren-Förderung zu sinkenden Börsenstrompreisen und Erlösen für Betreiber konventioneller (Reserve-)Kraftwerke. ${ }^{14}$

10 Vgl. M. Gottstein, S. Skillings: Über Kapazitätsmärkte hinausdenken: Flexibilität als Kernelement, in: Energiewirtschaftliche Tagesfragen, 62. Jg. (2011), H. 11, S. 18-25; T. Beckers, A. Hoffrichter: Grundsätzliche und aktuelle Fragen des institutionellen Stromsektordesigns Eine institutionenökonomische Analyse zur Bereitstellung und Refinanzierung von Erzeugungsanlagen mit Fokus auf FEE, Berlin 2014; M. Reeg: Entwurf eines ganzheitlichen Marktdesigns für hohe Anteile erneuerbarer Energien, Diskussionspapier, Nr. 01/2014, Systemanalyse und Technikbewertung, DLR-Institut für Technische Thermodynamik, Stuttgart 2014.

11 Vgl. ENTSO-E: Scenario Outlook and Adequacy Forecast 2013-2030, European Network of Transmission System Operators for Electricity (ENTSO-E), Brüssel 2013.

12 Vgl. M. Gottstein, S. Skillings, a.a.O.; M. Reeg, a.a.O.

13 Vgl. ebenda.

14 Vgl. T. Traber, C. Kemfert: Gone with the wind? - Electricity market prices and incentives to invest in thermal power plants under increasing wind energy supply, in: Energy Economics, 33. Jg. (2011), Nr. 2, S. $249-256$.
Ob und wie die Akteure im Stromversorgungssystem auf diese Herausforderungen reagieren und ihre Entscheidungen an veränderte Rahmenbedingungen anpassen, hängt jedoch auch maßgeblich von der grundlegenden Funktionsfähigkeit des Strommarktes ab. Prinzipiell honoriert der Strommarkt in seiner gegenwärtigen Ausgestaltung sowohl Investitionen in neue Kraftwerke und Speicher als auch Anpassungen der Stromnachfrage. Der Markt für Regelenergie zur Sicherung der kurzfristigen Systemstabilität belohnt dabei zusätzlich besonders flexible Optionen zum Ausgleich von Angebot und Nachfrage. Langfristige Signale zum Bau von Kraftwerken oder Speichern werden theoretisch durch die Terminmärkte gesendet. $^{15}$ Die Funktionsfähigkeit des Strommarktes kann jedoch aufgrund verschiedener Ursachen zusätzlich beeinträchtigt werden. Marktmacht, kurzfristiges Renditestreben oder der imperfekte Umgang mit Marktunsicherheiten - insbesondere im Zusammenhang mit asymmetrischer Information - können die Effizienz der Entscheidungen der Marktakteure beeinträchtigen. Unter den gegenwärtigen Rahmenbedingungen reagieren auch die Stromverbraucher nur begrenzt auf kurzfristig veränderte Knappheitssignale der Strompreise, zumal diese oftmals gar nicht bis zum Endverbraucher durchgereicht werden. Zudem können die regulatorischen Rahmenbedingungen - beispielsweise politisch gesetzte Preisobergrenzen oder langwierige Genehmigungsverfahren - Investitionen bremsen. ${ }^{16}$ Damit wird deutlich, dass die Diskussion um Versorgungssicherheitsprobleme nicht allein auf die Ausgestaltung der Energiewendeinstrumente zur Forcierung des Erneuerbare-Energien-Ausbaus fokussieren kann, sondern alle möglichen Ursachen in den Blick nehmen muss.

Ob es dabei im Zuge der Strom-Energiewende tatsächlich zu Versorgungsengpässen kommen wird, bleibt weiterhin höchst unsicher. Typische Indikatoren weisen darauf hin, dass prinzipiell genügend Kapazitäten verfügbar sind, um die maximal zu erwartende Stromnachfrage zu decken. So fällt die Leistungsbilanz, also der Saldo aus (gesicherter) Kraftwerksleistung und der maximalen Stromnachfrage gegenwärtig und wohl auch

15 Vgl. BMWi: Ein Strommarkt für die Energiewende, Diskussionspapier des Bundesministeriums für Wirtschaft und Energie (Grünbuch), Berlin 2014; Frontier Economics: Strommarkt in Deutschland - Gewährleistet das derzeitige Marktdesign Versorgungssicherheit?, Frontier Economics, London 2014.

16 Für einen Überblick zu möglichen Ursachen vgl. P. Cramton, A. Ockenfels: Economics and Design of Capacity Markets for the Power Sector, in: Zeitschrift für Energiewirtschaft, 36. Jg. (2012), Nr. 2, S. 13134; Frontier Economics, a.a.O. 
Abbildung 1

Installierte Leistung der Stromerzeugung und die Spitzenlast der Stromnachfrage in Deutschland bis 2035

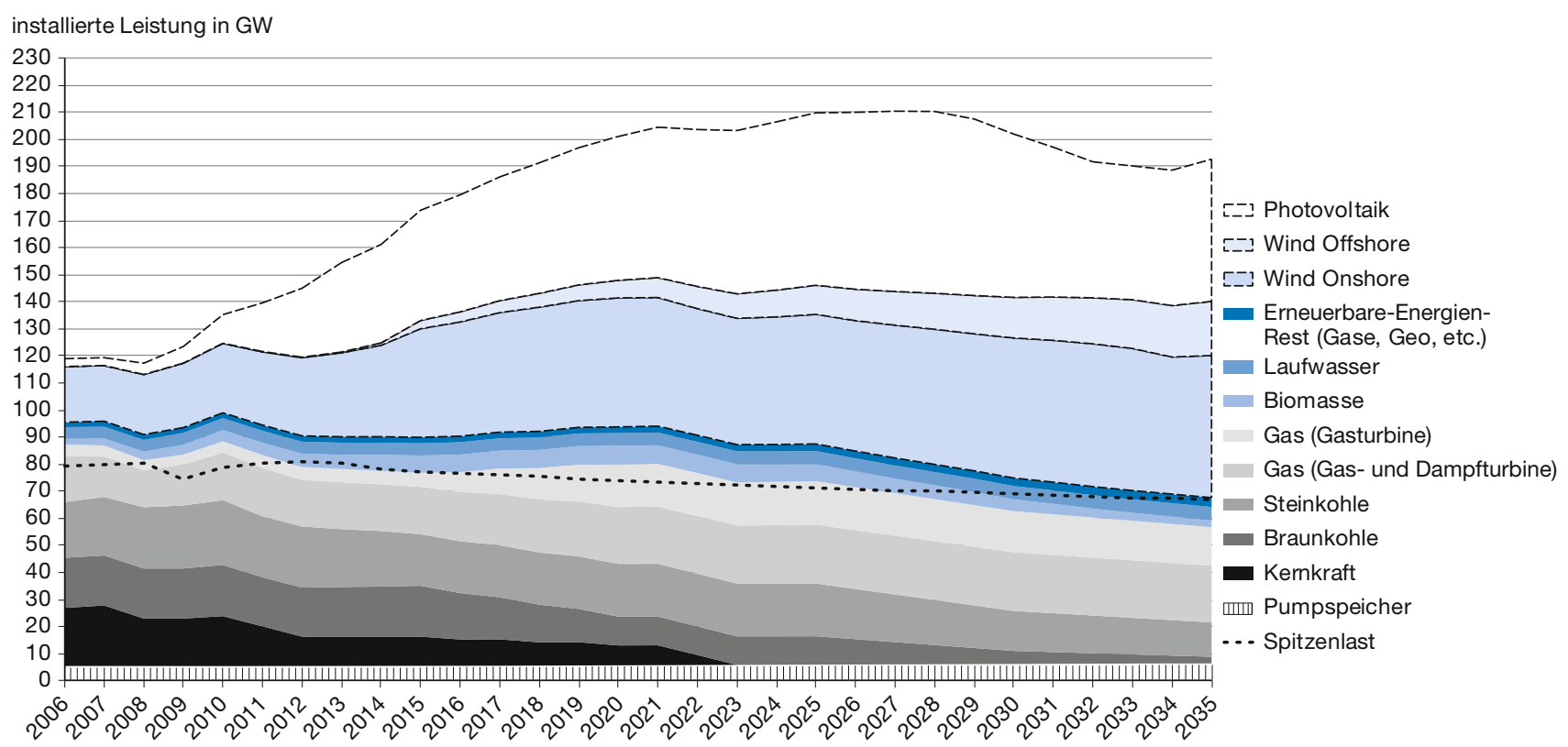

Für die Beurteilung der Versorgungssicherheit ist insbesondere zu berücksichtigen, welcher Anteil der installierten Leistung gesichert, also ständig verfügbar, ist. Für konventionelle Kraftwerke liegt die gesicherte Leistung nahe an der installierten Leistung. Für Windkraft und Photovoltaik (gestrichelte Bereiche der installierten Leistung) beträgt die gesicherte Leistung jedoch deutlich unter $10 \%$ der installierten Leistung. Die Abbildung illustriert also, dass die gesicherte Leistung mittelfristig weiter über der Spitzenlast der Stromnachfrage liegen wird. Die Abbildung vernachlässigt Aspekte der regionalen Verfügbarkeit und der Flexibilität der gesicherten Erzeugungskapazität, die zusätzlich für die Sicherung der Stromversorgung wichtig sind.

Quelle: Eigene Abbildung, J. Nitsch et al.: Langfristszenarien und Strategien für den Ausbau der erneuerbaren Energien in Deutschland bei Berücksichtigung der Entwicklung in Europa und global, Bundesministerium für Umwelt, Naturschutz und Reaktorsicherheit (BMU), 2012, http://elib.dlr.de/76043/; PLATTS: World Electric Power Plants Database, McGraw Hill Financial, 2010 und 2015.

für die nächsten Jahre positiv aus (vgl. Abbildung 1). ${ }^{17}$ Zudem bestehen auch Überkapazitäten in anderen europäischen Ländern, aus denen Deutschland zusätzlichen Strom importieren könnte. Einschränkungen gibt es möglicherweise jedoch beim kurzfristigen Ausgleich von Stromangebot und -nachfrage, also der Flexibilität des Stromversorgungssystems. Darauf weisen unter Umständen durch die Bundesnetzagentur untersagte Stilllegungen von Kraftwerken und der Abruf der Netzreserve hin. ${ }^{18}$ Jedoch sind diese Maßnahmen bislang

17 Vgl. auch Bundesnetzagentur, Bundeskartellamt: Monitoringbericht 2015, Bonn 2015, S. 68, http://www.bundesnetzagentur.de/ SharedDocs/Downloads/DE/Allgemeines/Bundesnetzagentur/ Publikationen/Berichte/2015/Monitoringbericht_2015_BA.pdf?_blob=publicationFile $\&=4$.

18 So stieg der Netzreservebedarf von 3,636 MW 2014/15 auf 7,515 GW 2015/16, vgl. ebenda, S. 66 f. eher Ausdruck von Netzengpässen als von zu geringen Erzeugungskapazitäten. ${ }^{19}$

3. Versorgungssicherheit sollte nicht um jeden Preis gewährleistet werden! Für eine angemessene Bewertung von Maßnahmen zur Gewährleistung von Versorgungssicherheit müssen zudem alle gesellschaftlich relevanten Kriterien und Ziele berücksichtigt werden. Zweifellos sollte ein bestimmtes Niveau an Versorgungssicherheit zu möglichst geringen Kosten gewährleistet werden. Vor diesem Hintergrund muss der regulatorische Rahmen so gewählt werden, dass die aus volkswirtschaftlicher Sicht günstigsten Investitionen vorgenommen werden - ganz gleich, ob diese nun die Errichtung neu-

19 Vgl. Deutsches Zentrum für Luft- und Raumfahrt (DLR), Institut für Energiewirtschaft und Rationelle Energieanwendung (IER): Szenarien der Versorgungssicherheit in Deutschland und Süddeutschland, Kurzstudie im Auftrag des Ministeriums für Umwelt und Energiewirtschaft Baden-Württemberg (UMBW), Stuttgart 2016, https:// um.baden-wuerttemberg.de/fileadmin/redaktion/m-um/intern/Dateien/ Dokumente/5_Energie/Versorgungssicherheit/160315_Szenarien_ der_Versorgungssicherheit_in_D_und_Sueddeutschland.pdf. 
er Kraftwerke, den Ausbau von Speichern und Netzen, Nachfragemanagement oder aber einen Mix aus diesen Optionen betreffen. Gleichzeitig müssen Maßnahmen zur Gewährleistung der Versorgungssicherheit in Einklang mit den politischen Zielen des Klimaschutzes, des Erneuerbaren-Ausbaus und der Energieeffizienz stehen. Zudem muss geprüft werden, wer etwaige Zusatzkosten einer höheren Versorgungssicherheit zu tragen hätte, welche Zusatzbelastungen also auf die privaten Haushalte und Unternehmen zukämen. Und nicht zuletzt müssen die Maßnahmen auch praktikabel sein - also mit vertretbarem administrativem Aufwand verbunden, in Anbetracht der diversen Unsicherheiten an veränderte Rahmenbedingungen und Wissensstände anpassbar sowie mehrheitsfähig und zugleich resistent gegen Einflussnahme partikularer Interessen. Eine bloße Verengung der Debatte auf das Ziel Versorgungssicherheit kann den Anforderungen an eine nachhaltige Umgestaltung des Energiesystems nicht gerecht werden und den Transformationsprozess unter Umständen sogar behindern.

4. Versorgungssicherheit muss durch einen Instrumentenmix gewährleistet werden! Wie bereits angedeutet, können Beiträge zur Versorgungssicherheit durch eine Vielzahl von Maßnahmen, die bei den unterschiedlichen Akteuren im Stromversorgungssystem ansetzen, geleistet werden. Mögliche Optionen reichen von der Vollendung der Strommarktliberalisierung (um Marktmacht einzudämmen und Preisobergrenzen überflüssig zu machen) über regional differenzierte Großhandelspreise (um regionale Kapazitätsengpässe besser zu signalisieren) bis hin zu beschleunigten Genehmigungsverfahren für Infrastrukturneubauten. ${ }^{20}$ Im Bereich der Energiewendepolitik könnten verstärkt Anreize für eine bedarfsgerechtere Einspeisung von erneuerbaren Energien gesetzt werden. ${ }^{21}$ Eine grundlegende Ertüchtigung des Emissionshandels würde Investitions- und Einsatzentscheidungen im Kraftwerksbereich zudem wieder stärker zugunsten emis-

20 BMWi: Ein Strommarkt ..., a.a.O.; Frontier Economics, a.a.O.; A. Löschel, F. Flues, F. Pothen, P. Massier: Den Strommarkt an die Wirklichkeit anpassen - Skizze einer neuen Marktordnung, in: Energiewirtschaftliche Tagesfragen, 63. Jg. (2013), H. 10, S. 22-25.

21 E. Gawel, A. Purkus, K. Korte, P. Lehmann: Förderung der Markt- und Systemintegration erneuerbarer Energien - Perspektiven einer instrumentellen Weiterentwicklung, in: Vierteljahreshefte zur Wirtschaftsforschung, 82. Jg. (2013), H. 3, S. 123-136; E. Gawel, A. Purkus: Promoting the Market and System Integration of Renewable Energies through Premium Schemes - A Case Study of the German Market Premium, in: Energy Policy, 61. Jg. (2013), S. 599-609; P. Lehmann, E. Gawel: Die Förderung der erneuerbaren Energien nach der EEGReform 2014, in: Wirtschaftsdienst, 94. Jg. (2014), H. 9, S. 651-658; A. Purkus et al.: Der Beitrag der Marktprämie zur Marktintegration erneuerbarer Energien - Erfahrungen aus dem EEG 2012 und Perspektiven der verpflichtenden Direktvermarktung, in: Energiewirtschaftliche Tagesfragen, 64. Jg. (2014), H. 12, S. 8-16. sionsarmer und flexiblerer Gaskraftwerke verschieben. Darüber hinaus könnten gezielte Maßnahmen den Ausbau und Betrieb von Netzen, Speichern und Nachfragemanagement anreizen. Auch hier ist das denkbare Portfolio breit und reicht von Anpassungen bei der Anreizregulierung von Netzen bis hin zur Technologieund Forschungsförderung für Batteriespeicher und intelligente Stromnetze. ${ }^{22}$

Gerade unter dem Gesichtspunkt der allokativen Effizienz ist es geboten, Stromversorgung durch einen breiten Instrumentenmix zu sichern. Mit einem Maßnahmenbündel können die diversen Ursachen möglicher Versorgungsengpässe zielgerechter und kostengünstiger adressiert werden als etwa mit pauschalen Kapazitätszahlungen. Zudem brächten manche der Instrumente auch zusätzliche Vorteile neben einer verbesserten Versorgungssicherheit mit sich. So wäre etwa eine Verschärfung des Emissionshandels auch einem effektiveren Klimaschutz dienlich. Naturgemäß muss dabei auch in einem Instrumentenmix für die einzelnen Maßnahmen sorgfältig geprüft werden, welcher Grad staatlicher Intervention tatsächlich notwendig ist und inwieweit wettbewerbliche Elemente in den Mix integriert werden können. Angesichts der hohen regulatorischen Komplexität müssen die Maßnahmen im Verbund zudem sorgfältig aufeinander abgestimmt werden. Ebenso ist jedoch klar, dass einzelinstrumentelle Ansätze, die etwa primär auf eine Reform der Förderung erneuerbarer Energien oder die Einführung von Kapazitätszahlungen setzen, keine nachhaltigen Steuerungsoptionen darstellen können.

\section{Bewertung des „Strommarktes 2.0“}

Vor dem Hintergrund dieser Überlegungen kann die Entscheidung der Bundesregierung für einen „Strommarkt 2.0“, der mit einem breiten Maßnahmenpaket unterlegt werden soll, grundsätzlich positiv bewertet werden. Die im Vorfeld der Entscheidung immer wieder geforderte alternative Lösung, die Stromversorgung vorrangig durch völlig neue Kapazitätsmärkte zu sichern, hätte zum gegenwärtigen Zeitpunkt hohe Risiken mit sich bringen können. Kapazitätsmärkte hätten das Betriebskonzept für konventionelle Kraftwerke grundlegend verändert: Betreiber hätten fortan nicht mehr nur eine Vergütung

22 M. Achtnicht, R. Madlener: Factors Influencing German House Owners' Preferences on Energy Retrofits, in: Energy Policy, 68. Jg. (2014), S. 254-263; I. Kastner, E. Matthies: Implementing web-based interventions to promote energy efficiency behavior at organizations - a multi-level challenge, in: Journal of Cleaner Production, 62. Jg. (2014), Nr. 1, S. 89-97; K. Korte, E. Gawel: Stromnetzinvestitionen und Anreizregulierung - Problemfelder und Lösungsansätze, in: Wirtschaftsdienst, 95. Jg. (2015), H. 2, S. 127-134. 
je Megawattstunde erzeugten Stroms erhalten, sondern zusätzlich auch noch je Megawatt vorgehaltener Kapazität. Trotz der Bezeichnung „Markt“ im Titel hätte es sich dabei um einen massiven staatlichen Eingriff in den Strommarkt gehandelt, etwa weil die Menge und Qualität der vorzuhaltenden Kapazität administrativ festzulegen wären. $\mathrm{Ob}$ eine derartige staatliche Umsteuerung das Stromversorgungssystem tatsächlich in die richtige Richtung gelenkt hätte, wäre angesichts der bestehenden Überkapazitäten und Unsicherheit völlig unklar gewesen. In Anbetracht des massiven Branchendrucks wäre die Gefahr groß gewesen, dass gestrandete Investitionen in konventionelle Kraftwerke zusätzlich vergoldet und die notwendige Marktbereinigung und der Umbau des deutschen Kraftwerksparks weiter verlangsamt worden wären. Gleichzeitig hätten die neuen Zusatzzahlungen in Zukunft wohl nur schwerlich wieder abgeschafft werden können, auch wenn sie sich als überflüssig und nicht zielführend erwiesen hätten. Schließlich wäre zu erwarten, dass die zukünftigen Zahlungsempfänger ein großes Interesse an der Aufrechterhaltung der Zahlungen hätten und dieses Interesse auch politisch geltend machen würden.

Mit dem vorgeschlagenen Maßnahmenmix setzt die Bundesregierung primär auf die schrittweise Weiterentwicklung und Ertüchtigung der regulatorischen Rahmenbedingungen. Dabei nimmt sie alle Akteure auf der Angebotsund Nachfrageseite in den Blick und setzt insbesondere auf die Stärkung der Marktmechanismen. Dieser Ansatz ist prinzipiell besser geeignet, die diversen Ursachen möglicher Versorgungsengpässe gezielt zu adressieren. $\mathrm{Ob}$ der Instrumentenmix genug Wirkung entfalten kann, bleibt freilich abzuwarten. So wirken insbesondere die vorgeschlagenen Maßnahmen in den Bereichen Netze, Speicher und Nachfragemanagement noch recht vage und erratisch und bedürfen voraussichtlich weiterer Anpassungen und Präzisierungen. Es muss dabei jedoch klar sein, dass Versorgungssicherheit aufgrund der bestehenden Unsicherheiten in jedem Fall nur durch kontinuierliches politisches Lernen und Nachsteuern in nachhaltiger Weise gesichert werden kann.

Dass sich die Bundesregierung dazu entschlossen hat, dem Marktmechanismus doch nicht vollständig zu vertrauen, sondern durch zusätzliche Kraftwerksreserven für eine Absicherung zu sorgen, ist ebenfalls nachvollziehbar. Die politischen (und wirtschaftlichen) Risiken etwaiger Stromausfälle wären zu groß. Dabei können die Reserven ordnungspolitisch als minimalinvasive Maßnahme zur Absicherung der Stromversorgung verstanden werden, die nur ausgewählte Kraftwerke betrifft - wohingegen etwa ein Kapazitätsmarkt flächendeckend Zusatzzahlungen verteilt hätte. Natürlich ist auch eine Kraftwerksreserve nicht frei von Problemen, wie die Monopolkommission richtig anmerkt. Marktmacht und politische Einflussnahme können die Kosteneffizienz der Reserve infrage stellen. Derartige Probleme stellten sich jedoch in ganz ähnlicher Weise auch bei weiterführenden Kapazitätsmechanismen.

Als eindeutig falsch muss jedoch die Entscheidung betrachtet werden, alte Kohlekraftwerke in einer „EdelKlimareserve" zu parken. Auf diese Weise können zwar möglicherweise nationale Klimaziele kurzfristig erreicht werden. Am globalen Ausstoß von Treibhausgasen ändert das jedoch nichts, da die Reduktionen durch den Mechanismus des europäischen Emissionshandels an anderer Stelle, d.h. außerhalb des Stromsektors und/oder außerhalb Deutschlands, notwendigerweise kompensiert werden. Zudem behindert diese Maßnahme einmal mehr den notwendigen Strukturwandel in der deutschen Stromerzeugung und damit auch die Erreichung der langfristigen nationalen Klimaziele. Der ursprüngliche Vorschlag eines mit dem Emissionshandel zu vereinbarenden „Klimabeitrages" hatte im Vergleich dazu deutlich mehr Reiz, wenn er auch ebenfalls theoretisch nicht so effizient gewesen wäre wie eine Ertüchtigung des Emissionshandels selbst. ${ }^{23}$

\section{Langfristige Energiepolitik in der Zwickmühle}

Insgesamt begibt sich die Bundesregierung mit ihrer Doppelstrategie aus „vertrauender" Stärkung und „misstrauender" Absicherung des Strommarktes aber in eine politische Zwickmühle. Das signalisierte Marktvertrauen wirkt umso stärker als Investitionsanreiz für private Akteure, je weniger der Regulierer selbst die Funktionsfähigkeit des Marktes in Zweifel zieht und je weniger wahrscheinlich weitere politische Eingriffe in der Zukunft sind. Durch die Absicherung des Marktes mit zusätzlichen Kraftwerksreserven werden aber gerade solche Zweifel genährt - auch wenn dieser Schritt in Anbetracht der herrschenden Unsicherheit nachvollziehbar ist. Je höher die Reserve ausfällt, desto weniger glaubhaft kann Vertrauen in den Markt vermittelt werden. Hinzu kommt, dass die Bundesregierung auch weitere regulatorische Anpassungen in der Zukunft nicht glaubhaft ausschließen kann. Neue Sachlagen können weitere regulatorische Schritte erfordern.

Zudem stellt sich - wie die Monopolkommission zu Recht anmerkt - die grundsätzliche Frage, ob die Preisspitzen politisch wirklich durchgehalten werden können. Zwar gehen Studien davon aus, dass extreme Preisspitzen zwischen 500 Euro und 3000 Euro pro Megawattstun-

23 Vgl. E. Gawel, S. Strunz: Ist ein nationaler „Klimabeitrag“ für die Kohlekraft sinnvoll - und wenn ja, welcher?, in: ifo-Schnelldienst, 68. Jg (2015), H. 14, S. 8-11. 
de nur in 20 bis 50 Stunden pro Jahr auftreten müssten, um beispielsweise Investitionen in Gaskraftwerke lohnend zu machen. Dies war beispielsweise in den Jahren 2005 bis 2007 der Fall, in denen in ca. 5 GW Gaskraftwerksleistung investiert wurde. ${ }^{24}$ Doch selbst derart seltene Preisspitzen können, der Aufmerksamkeitslogik von Politik und Medien folgend, bereits Forderungen nach Preisobergrenzen laut werden lassen, wie seinerzeit geschehen - zumal schwer zu unterscheiden sein dürfte, ob Preisspitzen wirklich Kapazitätsengpässe oder eher die Ausnutzung von Marktmacht abbilden. ${ }^{25} \mathrm{Ob}$ diese punktuellen Preisspitzen tatsächlich risikoaverse Betreiber mit rationalen Erwartungen zu den gewünschten Investments veranlassen, bleibt offen - insbesondere, wenn sie darauf spekulieren dürfen, dass Attentismus in Zukunft doch mit Extrazahlungen belohnt werden könnte. Daher ist unklar, ob die Politik dem Lobbydruck der Energiewirtschaft nach zusätzlichen Kapazitätszahlungen dauerhaft standhalten kann, da das Damoklesschwert drohender Blackouts stets über den politischen Entscheidungen schweben wird. Mithin muss zurzeit offen bleiben, ob das Maßnahmenpaket des „Strommarktes 2.0“ in der Praxis angemessene Investitionen der privaten Marktakteure zur Sicherung der Stromversorgung anreizen wird, selbst wenn es theoretisch dazu geeignet scheint.

\section{Fazit: Keine Ideallösungen vorhanden}

Wie kann die deutsche Energiepolitik mit dieser Zwickmühle umgehen? Der Vorschlag der Monopolkommission, den abgesicherten „Strommarkt 2.0“ in Anbetracht der Unwägbarkeiten gleichsam als „Testphase“ zu annoncieren, ist jedenfalls polit-ökonomisch kaum zielführend. Eine solche Ankündigung würde die regulatorische Unsicherheit zusätzlich erhöhen und die Energiebranche noch

24 Bundesnetzagentur: Kraftwerksliste, 2015, http://www.bundesnetzagentur.de/EN/Areas/Energy/Companies/SecurityOfSupply/ GeneratingCapacity/PowerPlantList/PubliPowerPlantList_node.html.

25 Vgl. C. Weber, P. Vogel: Marktmacht in der Elektrizitätswirtschaft Welche Indizien sind aussagekräftig, welche Konsequenzen adäquat?, in: Energiewirtschaftliche Tagesfragen, 57. Jg. (2007), H. 4, S. 20-24. expliziter dazu einladen, den „Strommarkt 2.0“ scheitern zu lassen. Noch größer wäre dann der Anreiz, in einer solchen Testphase durch strategische Zurückhaltung bei Stromerzeugung und Investitionen möglichst oft die Reserve zu aktivieren - und damit den Weg für einen für diesen Fall faktisch in Aussicht gestellten umfassenderen Kapazitätsmarkt zu ebnen. Genauso wenig hülfe es, die skizzierten politischen Unsicherheiten unerwähnt zu lassen. Rationale Marktakteure würden die Möglichkeit weiterer politischer Anpassungen trotzdem antizipieren und ihr Investitionsverhalten entsprechend anpassen. Mögliche Alternativen zum jetzt eingeschlagenen Weg des „Strommarkt 2.0“, allen voran der Einsatz von Kapazitätsmärkten schon zum jetzigen Zeitpunkt, brächten jedoch unter Umständen noch größere Risiken mit sich.

Somit stellt sich die Frage, ob es überhaupt eine sinnvolle Alternative zum eingeschlagenen Weg des „Strommarktes 2.0“ gibt. Dem Grundkonflikt zwischen Stärkung des Marktvertrauens und (misstrauender) staatlicher Absicherung zur Gewährleistung der Stromversorgung kann jedenfalls kaum aus dem Wege gegangen werden. Das ist jedoch kein Grund, vor den Herausforderungen der Versorgungssicherheit bereits jetzt durch vorauseilende flächendeckende Zahlungen für gesicherte Leistung zu kapitulieren oder gar die Strom-Energiewende infrage zu stellen. Lehrbuchhafte Ideallösungen sind nicht verfügbar und Zielkonflikte unvermeidbar. Vor diesem Hintergrund erscheint der Versuch sinnvoll und pragmatisch, etwaige Versorgungssicherheitsprobleme zunächst durch einen abgesicherten „Strommarkt 2.0“ zu lösen. Der einzige offensichtliche Makel im Maßnahmenprogramm bleibt die Kohle-Klimareserve. In jedem Fall war es jedoch zum gegenwärtigen Zeitpunkt die volkswirtschaftlich richtige Entscheidung, die Einführung umfassender Kapazitätsmärkte abzuwehren. Gleichfalls ist es wahrscheinlich, dass die Debatte um den richtigen Weg zur Sicherung der Stromversorgung in den nächsten Jahren regelmäßig wieder auf die politische Tagesordnung gesetzt wird und weitere politische Nachjustierungen folgen werden.

Title: Security of Power Supply With High Shares of Volatile Renewables: Does the Power Market Reform Deliver?

Abstract: Recently, concerns have been rising that the energy transition may impair the security of power supply in Germany by increasing the risk of shortages and black-outs in the long run. While power generation from volatile wind and solar energy has grown rapidly, incentives to invest in conventional back-up capacity have diminished. To respond to these concerns, the German government has adopted an energy policy reform package to strengthen the regulatory framework for the power market. But is this package appropriate to sustainably safeguard the German power supply?

JEL Classification: L11, L51, L94, Q41, Q48 